\title{
Reducing Power Consumption in LTE Data Scheduling with the Constraints of Channel Condition and QoS
}

\author{
Li-Ping Tung ${ }^{1}$, Ying-Dar Lin ${ }^{2}$, Yu-Hsien Ku르 ${ }^{2}$ Yuan-Cheng Lai ${ }^{3}$, and Krishna M. Sivalingam ${ }^{4}$ \\ ${ }^{1}$ Intelligent Information and Communications Research Center, National Chiao Tung University, Taiwan \\ ${ }^{2}$ Department of Computer Science, National Chiao Tung University, Taiwan \\ ${ }^{3}$ Department of Information Management, National Taiwan University of Science and Technology, Taiwan \\ ${ }^{4}$ Department of Computer Science and Engineering, Indian Institute of Technology Madras, Chennai, India \\ lptung@nctu.edu.tw, \{ydlin, yu0056.cs00g\}@cs.nctu.edu.tw, laiyc@cs.ntust.edu.tw, skrishnam@cse.iitm.ac.in
}

\begin{abstract}
GPP has developed the Discontinuous Reception (DRX) power saving mechanism, which periodically turns off the radio interface to reduce power consumption. However, packets cannot be received during the on period, as a result that the Quality of Services (QoS) may be violated. In addition, channel condition may be unstable during the transmission, so the optimal DRX parameters should also be dynamically adjusted. In this paper, we propose the Dynamic Scheduling with Extensible Allocation and Dispersed Offsets (DXD) scheme, which is composed of two algorithms, DRX parameter decision algorithm, to determine the DRX period by considering channel condition and QoS constraints, and DRX-aware scheduling algorithm, to determine whether to extend the on duration so that QoS would not be affected by DRX. Simulation results demonstrate that our DXD approach can reduce power consumption up to $96.9 \%$ compared to no DRX scheme and guarantee QoS as good as no DRX scheme.
\end{abstract}

\section{INTRODUCTION}

To save the energy of mobile devices, 3GPP LTE has defined the discontinuous reception (DRX) scheme to allow devices to turn off their radio interface and go for a sleep state for a length of time, while staying connected to the network, thereby reducing the power consumption when there is no data transmission. Nevertheless, if packets arrive at sleep state, these packets would pose unexpected delay, which may affect their Quality of Service (QoS) requirements. Thus, how to determine DRX parameters to reduce the power consumption and guarantee QoS simultaneously is still an open issue.

Channel condition should be also considered when determining the DRX parameters. The UE uses channel quality indicator (CQI) [1] to report channel condition for eNB, to decide the modulation and coding scheme (MCS) level. When channel condition is good, a higher MCS is assigned which means the UE can finish its transmission with a higher transmission rate within a shorter period. Thus, the UE can turn off its radio interface for a longer period for power saving. In contrast, when channel condition is bad, a lower MCS, which has a lower transmission rate, is assigned. Thus, the UE needs to extend the on duration in order to meet the QoS requirements. Therefore, CQI information should be considered when determining optimal DRX parameters.

Previous studies have investigated how to dynamically adjust the DRX parameters based on channel condition [2, 3].
A multi-threshold adaptive DRX mechanism [2] is proposed, where UEs are divided into several states according to their channel condition. UEs with better channel condition will be configured with lower power consumption parameters, and vice versa. In [3], DRX parameters are adjusted depending on the system load and the channel variation to improve power saving efficiency. For UEs with slow-varying channel, shorter DRX inactivity timer is used. However, the above work does not consider the QoS features, such as packet delay, packet loss rate, and required data rates.

In [4] and [5], the work considers the QoS requirements with multiple traffics when determining the DRX parameters. However, the channel condition is not taken into consideration. In [4], DRX parameters are configured based on delay requirement and power saving constraint. The proposed algorithm optimizes one of these two performance metrics while satisfying a pre-defined level of performance guarantee for the other. A traffic-based DRX cycles adjustment (TDCA) [5] scheme is proposed to improve power saving. It employs the partially observable Markov decision process (POMDP) to conjecture the present traffic status for DRX parameters selection while the packet delay constraint can still be satisfied.

DRX operations should be also taken into consideration when applying the scheduling algorithm [6]. The consideration means that DRX parameters are introduced into the scheduling determinants, so as to reduce packet loss or packet delay caused by the sleeping process during DRX. A DRX-aware scheduling scheme [6] is proposed to reduce the packet loss rate due to DRX sleep. The scheduler takes inactivity timer into consideration. If inactivity timer is smaller, the UE would be given a higher priority in order to meet delay requirements. In [7], the Three-Stage (TS) scheme determines DRX parameters by considering the QoS requirements for IoT (Internet of Things) applications. They also propose DRX aware scheduling algorithm. However, there are some differences between TS and our proposed work. First, the TS scheme only considers the applications with the strictest QCI to determine on duration even though the UE has to serve multiple services. Second, the TS scheme uses fixed DRX configuration which is based on the worst CQI report.

In this study, a novel DRX scheme, called Dynamic Scheduling with Extensible Allocation and Dispersed Offsets (DXD) scheme, is proposed to meet QoS requirements, reduce power consumption, and increase system capacity in the DRX 


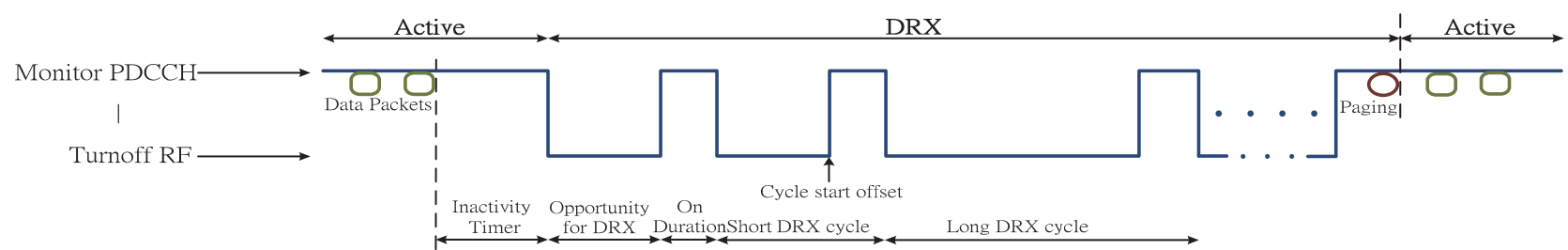

Fig. 1. Operation of DRX

mode. In order to reduce the power saving, the UE should turn off the radio interface as long as possible under the QoS constraints. The proposed scheme dynamically determines DRX parameters depending on QoS. On the other hand, when the UE turns on the radio interface, the buffered packets should be forwarded as soon as possible. Thus, we disperse on duration of UEs, so that each UE can fully utilize the bandwidth for resource efficiency. In addition, the proposed method includes a DRX-aware scheduler. It can determine whether or not to extend the on duration for QoS satisfaction. If system loading is high, extension may not improve QoS but only increases power consumption.

The rest of the paper is organized as follows. Section II presents the background of this work. In Section III, the problem statement is described. Then, the proposed Dynamic Scheduling with Extensible Allocation and Dispersed Offsets (DXD) scheme is presented in Section IV. In Section V, we present a comprehensive set of simulations, and the results are discussed in detail. Finally, Section VI gives the conclusions.

\section{BACKGROUND}

\section{A. Discontunuous Reception (DRX) Mechanism}

DRX is a power saving mechanism in LTE system. When applying the DRX scheme, the UE periodically turns off the radio interface to save power. During the period in which the UE is in the active state, the UE monitors Physical Downlink Control Channel (PDCCH) in order to check for incoming packets or paging signals, as shown in Figure 1. If paging signals or packets arrive, the UE stays active until no more packets are received for a period of time, i.e., Inactivity Timer. When the Inactivity Timer expires, the UE go to DRX sleep state. The DRX behavior can be configured by the DRX parameters including On Duration Timer, Opportunity for DRX, DRX Cycle, Inactivity Timer, and Cycle Start Offset. The DRX operation and parameters are illustrated in Figure 1.

On Duration denotes the time period in which the UE wakes up and monitors PDCCH. Opportunity for DRX denotes the time period in which the UE sleeps for power saving. DRX Cycle denotes the cycle by which On Duration repeats periodically. Inactivity Timer denotes the time period after the last scheduling that the UE should remain awake. If the UE does not receive any packet, this timer is decreased by one micro second. On the contrary, if the UE is scheduled, the Inactivity Timer is reset. Hence, this parameter provides means for the network to keep a UE awake beyond the On Duration when data is buffered. Cycle Start Offset denotes the start time of On Duration in each cycle. DRX parameters of all UEs are determined by the eNB. The eNB uses Radio Resource
Configuration (RRC) control signal to configure or update these DRX parameters. Then the UE updates its configuration after receiving the parameters from the eNB.

\section{B. QoS Class Identifier (QCI)}

As the wireless communication prevails, the traffic becomes more abundant and complicated. In order to meet the QoS requirements for different services, LTE specifically defines the QCI metric, which grades the QoS in nine classes. Each class contains quadruple, i.e., resource type, priority, packet delay budget, and packet error loss rate. Resource type denotes whether the traffic needs guaranteed bit rate (GBR) and its value if any. For priority, the smaller the number is, the higher priority the traffic owns. Packet delay budget puts the constraint on the transmission delay of each packet, and packet error loss rate claims on the acceptable error rate of traffic.

\section{PROBLEM STATEMENT}

We observed that the DRX parameter settings, QoS requirements, channel conditions of a UE are tightly coupled with each other. For example, the UE with good channel quality can use the higher MCS which means that the UE can transmit data with a higher transmission rate to finish the data transmission within a short period. Thus, the UE can turn off its radio interface for a longer period for power saving. In contrast, when channel condition is bad, a lower MCS, which has a lower transmission rate, is assigned. Thus, the UE needs to extend the on duration to meet the QoS requirements and consequently has higher power consumption. Therefore, how to configure DRX parameters is a critical problem.

In this study, we consider the LTE network of an eNB serving $N$ UEs. Each $U E_{i}, i=1, \ldots, N$, has $M_{i}$ application services, which are denoted as $S_{i, k}, k=1, \ldots M_{i}$. Each $S_{i, k}$ is assigned to a QoS class identifier (QCI) $Q C I_{i, k}$, including Packet Delay Budget $L_{i, k}(\mathrm{~ms})$ and Guaranteed Bit Rate $R_{i, k}$ (bps). Each $U E_{i}$ is configured with a set of DRX parameters $D_{i}$, including On Duration Timer $T O D_{i}(\mathrm{~ms})$, Opportunity for DRX $T O P_{i}(\mathrm{~ms})$, Long DRX Cycle $C L D_{i}(\mathrm{~ms})$, Inactivity Timer $T I A_{i}$ (ms), and Cycle Start Offset $C_{i} O_{i}(\mathrm{~ms})$. Furthermore, the channel quality indicator and power consumption are denoted as $C Q I_{i}$ and $P_{i}$, respectively.

The DRX parameters determination problem is how to allocate the resource blocks and determinate the DRX parameters $D_{i}$ for each $U E_{i}$, including $T O D_{i}, T O P_{i}, C L D_{i}, T I A_{i}$, and $C S O_{i}$, such that all QoS requirement $Q C I_{i}$ can be satisfied as like as in the DRX disenabling settings. In the meantime, the power consumption $P_{i}$ for each $U E_{i}$ could be reduced as much as possible. 


\section{DYNAMIC SCHEDULING WITH EXTENSIBLE ALLOCATION AND DisPERSED OFFSETS SCHEME}

In this study, a novel DRX scheme, called Dynamic Scheduling with Extensible Allocation and Dispersed Offsets (DXD), is proposed with the objectives of satisfying the QoS requirements, reducing the power consumption, and increasing the system capacity with DRX operations. As illustrated in Figure 2, the DXD scheme can be divided into two parts: 1) DRX parameter decision algorithm and 2) DRX-aware scheduling algorithm.

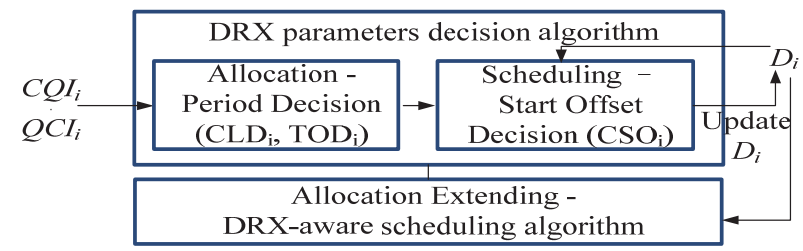

Fig. 2. Dynamic Scheduling with Extensible Allocation and Dispersed Offsets (DXD) scheme

First, we determine the optimal DRX period by considering channel condition and QoS constraints under the assumption that the UE can fully utilize the resource. Thus, the buffered packets can be transmitted within the on duration without violating their QoS requirements. Since the network resources are shared by multiple UEs, we need to disperse the on duration of UEs to let the UE fully utilize the bandwidth during the on duration and finish its transmission as soon as possible to go to sleep for more time. DRX parameters are also taken into consideration during scheduling process so that the active period can be dynamically extended or not. The detailed operations of DXD are described as follows.

\section{A. DRX Paremeters Decision Algorithm}

DRX parameter decision algorithm will be executed when one of the UEs needs to update its DRX parameters. DRX parameter decision algorithm includes two parts. In the first part, period decision method determines the allocation of on duration as well as DRX cycle for a UE. In the second part, the start offset decision method determines the cycle start offset to disperse the active period of UEs.

1) Allocation - Period Decision: When the $U E_{x}$ attaches to the eNB or detects the change of channel condition, period decision method need to update DRX cycle $C L D_{x}$ and on duration $T O D_{x}$. To maximize power saving and meet the strictest delay budget, DRX cycle is set as the minimum delay budget among all services for $U E_{x}$ as

$$
C L D_{x}=\min _{1 \leq k \leq M_{x}}\left\{L_{x, k}\right\} \text {. }
$$

In order to save power, $U E_{x}$ should turn off the circuit immediately after its all packets are transmitted. Therefore, on duration $T O D_{x}$ is set as the expected transmission time according to its channel quality indicator $C Q I_{x}$ as

$$
T O D_{x}=\left\lceil C L D_{x} \cdot \min \left\{\frac{\sum_{1 \leq k \leq M_{x}} R_{x, k}}{B\left(C Q I_{x}\right) \cdot N_{R B} / 0.0005}, 1\right\}\right]
$$

$T O D_{x}$ is obtained from multiplying the DRX cycle $C L D_{x}$ by the ratio of the sum of guaranteed bit rate $R_{x, k}$ to the available transmission rate. Available transmission rate is obtained based on current channel condition, thus we use $\mathrm{B}\left(C Q I_{x}\right)$ to denote the number of bits could be carried in one resource block (RB) by looking up the CQI table. Let $N_{R B}$ denote the number of RBs per slot, which length is $0.5 \mathrm{~ms}$. If the required data rate is larger than the available transmission rate, we set the on duration $T O D_{x}$ as the DRX cycle $C L D_{x}$.

2) Scheduling - Start Offset Decision: In order to improve radio resource efficiency in the on duration, we disperse the on duration of each UE by assigning different cycle start offsets. The UE can fully utilize the bandwidth during its on duration if its on duration does not overlap with others.

We maintain $N A=\left\{N A_{j}, 1 \leq j \leq C L C M\right\}$, in which $N A_{j}$ is the number of UEs which stays in the on duration at subframe $j$. Let CLCM denote the least common multiple (LCM) of all DRX cycles $C L D_{i}$. Since the DRX cycle $C L D_{x}$ will repeat multiple times within the CLCM time period, so we use $N R P_{x}=\left\{N R P_{x, k}, 1 \leq k \leq C L D_{x}\right\}$ in unit of $C L D_{x}$ to indicate the number of UEs which are active at the same offset in each cycle within the whole $C L C M$. So, we have $N R P_{x, k}$ as

$$
N R P_{x, k}=\sum_{\forall j,((j-1)} \sum_{\left.\bmod C L D_{x}\right)+1=k}, 1 \leq j \leq C L C M .
$$

Then, the cycle start offset $C S O_{x}$ can be found by

$$
C S O_{x}=\arg \min _{1 \leq k \leq C L D_{x}}\left\{\sum_{j=k}^{k+T O D_{x}-1} N R P_{x,\left((j-1) \bmod C L D_{x}\right)+1}\right\} \text {. }
$$

Equation (4) means that during the on duration of $U E_{x}$, the number of UEs which stay in the on duration is minimal within the whole $C L C M$ time period. Therefore, $U E_{x}$ can fully utilize the bandwidth during its on duration if it does not overlap with other UEs, finish its transmission as soon as possible, and go to sleep for more time.

However, we observe that the value of $N R P_{x}$ may not be sufficient to grasp the impact come from other UEs. Take Figure 3 as an example. Here, there are 3 UEs with different DRX cycles and UEs is active at the same time (case 1) or is active at different time (case 2). According to the definition of $N R P_{x}$, the value of $N R P_{x}$ in both cases are the same (i.e., $N R P_{x, 1}$ $=3$ and $N R P_{x, j}=0$, if $j \neq 1$ ). However, the impact from other UEs in case 1 is more than that in case 2 because all other UEs are active at the same time in case 1 . If $U E_{x}$ is also active at the same time, the network resource would be shared with other three UEs. On the other hand, UEs are active at the different time period in case 2 ; thus $U E_{x}$ only competes with one UE.

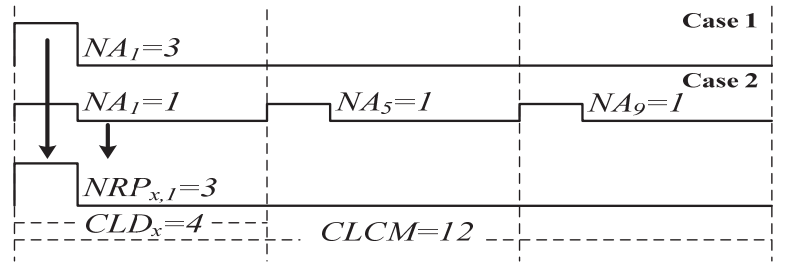

Fig. 3. The impact of other UEs for $U E_{x}$ with $C L D_{x}=4$ and $C L C M=12$ 
So we consider a weighted version of $N A$ as $N S=\left\{N S_{j} \mid N S_{j}=\left(N A_{j}\right)^{2}, 1 \leq j \leq C L C M\right\}$ in order to differentiate the degree of dispersion of UEs' on duration. A subframe with more active users has more impact on the start offset decision. Then, we have the new $N R P^{\prime}{ }_{x}$ as

$$
N R P_{x, k}^{\prime}=\sum_{\forall j,((j-1)} N S_{j}, 1 \leq j \leq C L C M .
$$

Then, the optimal cycle start offset $C S O_{x}$ can be found by

$$
C S O_{x}=\arg \min _{1 \leq k \leq C L D_{x}}\left\{\sum_{j=k}^{k+T O D_{x}-1} N R P_{x,\left((j-1) \bmod C L D_{x}\right)+1}^{\prime}\right\} \text {. }
$$

\section{B. Allocation Extending - DRX-aware Schedluing Algorithm}

The DRX aware scheduling in [7] dynamically extends the on duration to increase the satisfaction ratio on delay budget. DRX inactivity timer will be reset if the UE is scheduled. With this feature, the UE that will go for sleep at the next subframe is scheduled one RBG to extend the inactivity timer if RLC buffer is not empty. However, frequent extending the on duration will significantly increase power consumption, especially in the case of poor channel condition or high system load. The reason is that the system may not have sufficient radio resources to allocate to the UE in UE's extended on duration. Therefore, our scheme does not extend the on duration of UE if the system is unable to serve the UE. The formula of scheduling priority handling is described as follow.

$$
\begin{gathered}
p_{x}(t)=\left\{\begin{array}{cc}
p_{x}^{*}(t)+\alpha & , \text { if } T O D_{x} \leq 1, T I A_{x} \leq 1, \text { and } \rho_{\mathrm{x}}<\delta_{x}, \\
p_{x}^{*}(t) & , \text { otherwise }
\end{array}\right. \\
\rho_{x}=\sum_{\forall i, p_{i}^{*}(t)>p_{x}^{*}(t)} \frac{R_{i}(t)}{r_{i}(t)}, \text { and } \delta_{x}=\frac{T O P_{x}}{C L D_{x}},
\end{gathered}
$$

where $p_{x}^{*}(t)$ denotes priority of original scheduling which can be any scheduling scheme and $p_{x}(t)$ denotes our proposed priority handling function at subframe $t$ for $U E_{x}$. Let $\rho_{x}$ denote the total loading of active UEs whose priorities are higher than $U E_{x}$, and it is calculated by $R_{i}(t)$ and $r_{i}(t)$ which are average data rate and achievable data rate depending on $C Q I_{i}$ at subframe $t$, respectively. Let $\delta_{x}$ denote the ratio of opportunity for DRX to DRX cycle. So $\rho_{x} \geq \delta_{x}$ means that system loading is too high to serve $U E_{x}$ during the rest of time in its DRX cycle. Therefore, when $U E_{x}$ will turn off its radio interface at the next subframe (i.e., $T O D_{x} \leq 1, T I A_{x} \leq 1$ ) and the system load is less than the free resource from turn-off (i.e., $\rho_{x}<\delta_{x}$ ), the priority of UE will be increased by $\alpha$. Here, we let $\alpha>\max _{\forall i}\left\{p_{i}^{*}(t)\right\}$ to raise the priority of UE which need to be scheduled.

\section{Performance EVAluations}

We developed the proposed DXD scheme in NS-3 simulator [8]. The power consumption model in [9] is used to obtain the real power consumption of UE. Simulation configurations are summarized in TABLE I. If fixed DRX scheme is enabled, the default DRX parameters listed in TABLE I are used. Otherwise, the DRX parameters will be obtained from the proposed DXD approach. Performance metrics of measurement are: delay satisfaction ratio and power consumption. Delay satisfaction ratio indicates the percentage of packets that satisfy the delay budget.

TABLE I. SimUlation CONFIGURATIONS

\begin{tabular}{|c|c|c|}
\hline & $\begin{array}{c}\text { adaptability to } \\
\text { channel condition }\end{array}$ & system capacity \\
\hline Bandwidth & \multicolumn{2}{|c|}{$5 \mathrm{MHz}$} \\
\hline \# of UE & 8 & $5 \sim 30$ \\
\hline $\begin{array}{c}\text { default DRX } \\
\text { parameters }\end{array}$ & \multicolumn{2}{|c|}{$\begin{array}{c}(\mathrm{CLD}, \mathrm{TOD})= \\
(20 \mathrm{~ms}, 2 \mathrm{~ms}),(100 \mathrm{~ms}, 10 \mathrm{~ms}),(120 \mathrm{~ms}, 12 \mathrm{~ms}) \\
\mathrm{TIA}=3 \mathrm{~ms}, \mathrm{CSO}=0 \mathrm{~ms}\end{array}$} \\
\hline Scheduler & Proportional Fair Scheduler \\
\hline Services & $\begin{array}{c}\text { VoIP } \\
(\mathrm{QCI}: 1)\end{array}$ & $\begin{array}{c}\text { VoIP and video streaming } \\
\text { (QCI:4) }\end{array}$ \\
\hline $\begin{array}{c}\text { Channel } \\
\text { Condition }\end{array}$ & $\mathrm{CQI}=1 \sim 15$ & random \\
\hline
\end{tabular}

\section{A. Adaptability to Channel Conditions}

In order to observe the adaptability to channel conditions, we investigate the relationship between CQI and delay satisfaction ratio in Figure 4. A scheme has higher adaptability to channel condition means that it achieves high delay satisfactory ratio regardless of the channel condition.

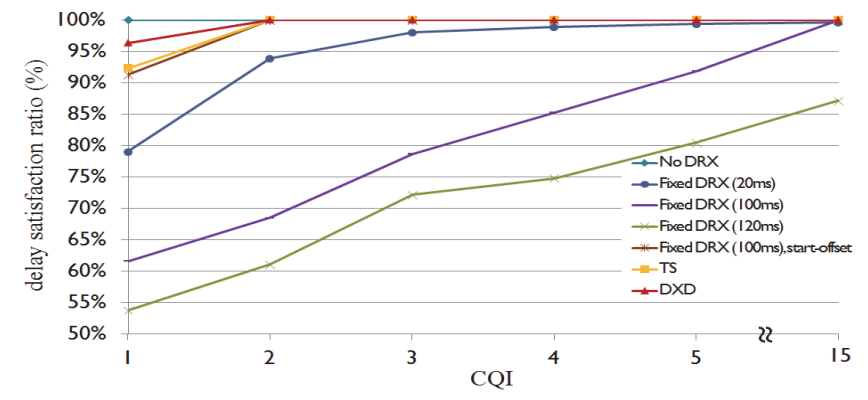

Fig. 4. Impact of channel conditions : delay satisfaction ratio

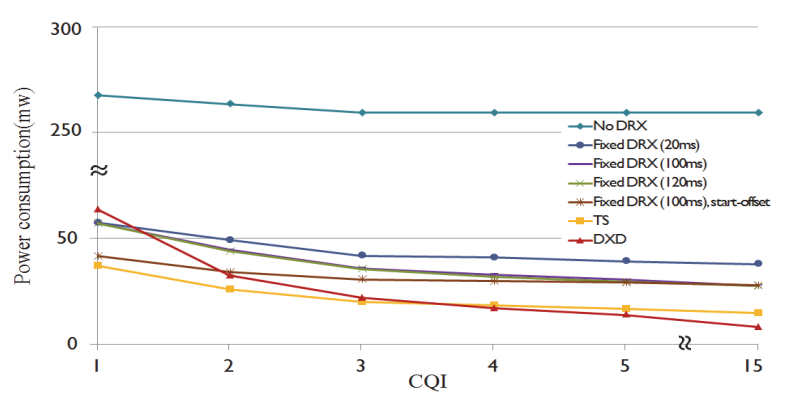

Fig. 5. Impact of channel conditions : power consuption

In Figure 4, "No DRX" label represents the case where DRX is not enabled. "No DRX" scenario has the highest adaptability to channel condition but also has the highest power consumption as shown in Figure 5. We observe the results of fixed DRX scheme with different lengths of DRX cycles. DRX scheme with longer DRX cycles has lower power consumption. Nevertheless, if the DRX cycle is longer than the delay budget of the services, delay budget cannot be satisfied even in the best channel condition. In addition, the results under the fixed DRX scheme with $100 \mathrm{~ms}$ DRX cycle shows that when CQI 
decreases, delay satisfaction ratio also decreases significantly. The reason is that when CQI decreases, the UE needs more radio resources to maintain the required data rate. If on durations of UEs are not interlaced, the performance will decrease seriously. Thus, it is obvious that delay satisfaction ratio can be significantly improved when start offset decision is applied. The proposed DXD scheme has the best satisfaction ratio even in a poor channel condition because it dynamically determines the on duration to finish the data transmission as soon as possible.

Compared with the TS scheme [7], the DXD scheme has better performance of the delay satisfaction ratio and the power consumption of the DXD scheme is lower than that in the TS scheme when CQI is between 4 to 15 . The reduction of power consumption compared to "No DRX" and TS is up to $96.9 \%$ and $45.7 \%$ respectively because the DXD scheme dynamically configures on duration according to channel condition. However, in the TS scheme, the on duration is determined by one packet transmission time with the worst channel condition. That is, the on duration in the TS scheme is not responsive to the channel condition. In addition, the on duration may be too small to satisfy the QoS in the worst channel condition.

\section{B. System Capacity Problem}

The effect of system loading upon QoS satisfaction and power consumption are shown in Figure 6 and Figure 7 receptivity. The performance of our proposed DXD approach is as good as that of "No DRX" scheme in a lightly-load environment. In the high system load environment, the performance of the DXD scheme is better than that of "No DRX" scheme. The number of satisfied UEs in the DXD scheme is more than that in "No DRX" scheme with PF scheduler. The reason is that when the system loading gradually increases, the delay satisfaction ratio could be improved by applying the proposed DRX-aware scheduling.

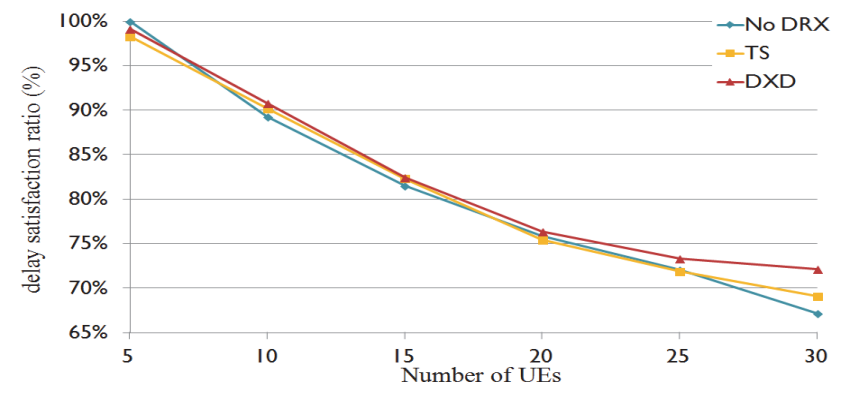

Fig. 6. Impact of number of UEs : delay satisfaction ratio

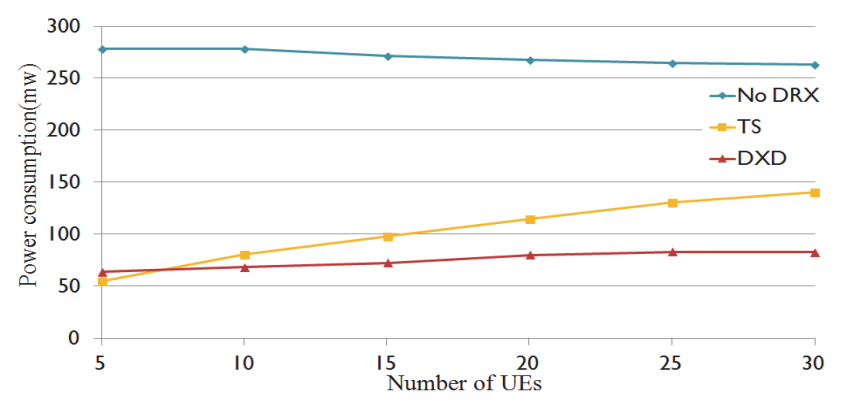

Fig. 7. Impact of number of UEs : power consumption
Figure 7 shows that the DXD scheme can save more power than the TS scheme when system loading is high. Power consumption can reduce up to $41.1 \%$. The main reason is that the TS scheme continues to extend the on period, but our DXD scheme determines whether it is necessary to extend or not. The DXD scheme only turns off UEs which are impossibly served according to system loading. So power can be saved without affecting the system performance.

\section{CONCLUSION}

In this paper, we propose the Dynamic Scheduling with Extensible Allocation and Dispersed Offsets (DXD) scheme to reduce power consumption and meet QoS in varied channel conditions. The DXD scheme is composed of period decision, start offset decision, and DRX-aware scheduling algorithm. First, to enhance the channel condition adaptability and satisfy multiple services in a UE, period decision method configures an optimal DRX period according to CQI and QCI of each service. Second, start offset decision method effectively disperses on duration of each UE, so that the radio resource can be utilized more efficiently. Third, the proposed DRX-aware scheduling dynamically extend the on duration of UEs to increase delay satisfaction ratio for the bursty traffic.

Simulation results show the outperformance of our DXD scheme. In the case of dynamic channel conditions, the delay satisfaction ratio is only slightly lower than that of "No DRX" scheme in poor channel condition. In the meantime, the power consumption can be reduced by $45.7 \%$ compared to the TS scheme in good channel condition. When the number of UEs increases gradually, the power consumption is reduced up to $41.1 \%$, compared to the TS scheme. In summary, our approach can effectively reduce power consumption and has better delay satisfaction ratio in varied channel conditions and high loading system.

\section{REFERENCES}

[1] 3GPP TS 36.213: "Technical Specification Group Radio Access Network; Evolved Universal Terrestrial Radio Access (E-UTRA); Physical layer procedures".

[2] Songtao Gao, Hui Tian, Jianchi Zhu, and Lan Chen, "A more powerefficient adaptive discontinuous reception mechanism in LTE," in Proc. VTC-2011 Fall,pp. 5-8, Sept. 2011.

[3] Liu Liu, Xiaoming She, and Lan Chen, "Multi-user and channel dependent scheduling based adaptive power saving for LTE and beyond system ," in Proc. APCC 2011, Oct. 31 2010-Nov. 32010.

[4] Satish Chandra Jha, Ali Taha Koc, and Rath Vannithamby, "Optimization of Discontinuous Reception (DRX) for Mobile Internet Applications Over LTE, ” in Proc. VTC 2012-Fall, 2012.

[5] Yu-Ping Yu and Kai-Ten Feng, "Traffic-Based DRX Cycles Adjustment Scheme for 3GPP LTE Systems," in Proc. VTC2012-Spring, 2012.

[6] Huang Bo, Tian Hui, Chen Lan, and Zhu Jianchi, "DRX-aware scheduling method for delay-sensitive traffic," IEEE Communications Letters, vol. 14, no. 12, pp. 1113-1115, December 2010.

[7] Jen-Jee Chen, Hung-Hsin Cheng, Hung-Hsin Cheng, and Yu-Chee Tseng, "An Energy-Efficient Sleep Scheduling With QoS Consideration in 3GPP LTE-Advanced Networks for Internet of Things," IEEE Journal on Emerging and Selected Topics in Circuits and Systems, vol. 3 , no. 1, pp.13-22, March 2013.

[8] "Network Simulator 3," available at: http://www.nsnam.org/.

[9] Nokia, R2-071285, "DRX parameters in LTE," March 2007 